\title{
Saúde bucal e uso dos serviços odontológicos em função do Índice de Necessidades em Saúde: São Paulo, 2008
}

\author{
Oral health and access to dental care services \\ in relation to the Health Necessities Index. São Paulo, Brazil, 2008
}

Simone Rennó J unqueira ${ }^{1}$

Antônio Carlos Frias ${ }^{1}$

Celso Zilbovicius ${ }^{1}$

Maria Ercilia de Araujo ${ }^{1}$

${ }^{1}$ Departamento de Odontologia Social, Faculdade de Odontologia, Universidade de São Paulo. Av. Prof. Lineu Prestes 2227, Cidade Universitária. 05508-900 São Paulo SP. srj@usp.br
Abstract The Health Department of São Paulo, Brazil, has developed a Health $\mathrm{N}$ ecessities Index $(\mathrm{HNI})$ to identify priority areas for providing health assistance. In 2008, a survey of the status of oral health was conducted. The objective of this ecological study was to analyze the status of oral health in relation to the H NI. The variables, stratified by the age of 5, 12 and 15 years old were: percentage of individuals with difficulty of access to dental care services; DM FT and DM FS; prevalence of the need for tooth extraction and treatment of dental caries. Data were analyzed for the $25 \mathrm{H}$ ealth Technical Supervision Units(HTS). The Statistical Covariance Test was used as well as the Pearson correlation coefficient and linear regression model. A positive correlation was observed between high scores of the HNI and difficulty of access to services. In the HTS with high scores of $\mathrm{HNI}$ a higher incidence of dental caries was observed, a greater need for tooth extractions and low caries-free incidence. In order to improve health conditions of the population it is mandatory to prioritize actions in areas of social deprivation. Key words $\mathrm{H}$ ealth service needs and demands, $H$ ealth service accessibility, Oral health, DM FT index
Resumo A Secretaria M unicipal deSaúdedeSão Paulo desenvolveu o Índice de Necessidades em Saúde (INS) para identificar áreas prioritárias para a oferta de serviços. Em 2008 realizou um Levantamento das Condi ções de Saúde Bucal. Pre tende-se, neste estudo ecológico, analisar o perfil de saúde bucal em relação ao INS. As variáveis, estratificadas para as idades de 5, 12 e 15 anos, foram: porcentagem de indivíduos com dificuldade no acesso ao serviço odontológico; média do ceod e CPOD; prevalência da necessidade de extração e de livres de cárie. As informações foram analisadas para as 25 Supervisões Técnicas de Saúde (STS). Empregou-se o teste estatístico de covariância, o coeficiente de correlação de Pearson e o modelo de regressão linear. O bservou-se uma correlação positiva entre maiores INS e a dificuldade de acesso aos serviços. N as STS com maiores INS houve maior experiência de cárie, maior necessidade de exodontias e menor prevalência de livres de cárie. Reforça-se a necessidade de priorizar as áreas de privação social para meIhorar a condição de saúde da população.

Palavras-chave Necessidades edemandas deserviçosdesaúde, Acesso aos serviços de saúde, Saúde bucal, Índice CPO 
Introdução

Embora a prevalência ea severidadeda cáriedentária tenham diminuído entre criançase adolescentes brasileiros nas duas últimas décadas do século passado, persistem as dificuldades para assegurar tratamento dentário às pessoas afetadas ${ }^{1}$.

0 último levantamento epidemiológico em saúde bucal de âmbito nacional, conhecido como SB-Brasil $2003^{2}$ mostrou uma distribuição heterogênea da doença, registrando diferenças entre as regiões e os grupos sociais. 0 estudo constatou que a experiência da doença foi mais severa nos grupos populacionais mais submetidos à privação social ${ }^{3}$.

Diversos levantamentos têm sido realizados em nível local, pois permitem delinear o perfil ecológico da cárie dental e avaliar a tendência de comportamento dessa doença. M ais do queisso, orientam o planejamento e a organização dos serviços de saúde bucal para que sejam direcionados em função das necessidades, pautados nos princípios da equidadee da integralidade do Sistema Ú nico de Saúde.

O papel do território na reprodução das iniqüidades em saúde superou a concepção natural do espaço apenas como um ambiente inerte e passivo etem sido referenciado como espaço geográfico social mente construído e relevante para a compreensão dos fenômenos envolvidos no processo saúde doença4.

Assim, a Coordenação de Epidemiologia e Informação (CEInfo) da Secretaria M unicipal de SaúdedeSão Paulo (SM S-SP) desenvolveu o Índice deN ecessidades em Saúde (INS), usado para o acompanhamento dos resultados da atuação da própria Secretaria nas prioridades do Sistema Ú nico de Saúde(SUS), para possibilitar a identificação de áreas e grupos populacionais a serem priorizados para a oferta de serviços de saúde na cidade.

As necessidades contempladas no índice refletem a demanda potencial pelos serviços de saúdee são expressas nas condições demográficas, epidemiológicas e sociais da população residentenos 96 Distritos Administrativos (DA) da cidade, que estão sob a supervisão de 25 Supervisões Técnicas de Saúde (STS) que, por sua vez, estão distribuídas nas cinco Coordenadorias Regionais de Saúde ${ }^{5}$.

Ao IN S não são agregadas informações em saúde bucal, mas sabe-se que estas são influenciadas por condições demográficas e socioeconômicas. Precárias condições de vida dificultam a priorização dos cuidados em saúde, inclusive os bucais ${ }^{6}$.
Em 2008 a Área Técnica de Saúde Bucal da SM S-SP realizou um Levantamento das Condições de Saúde Bucal para as idades de 5, 12 e 15 anos deidade, com inferência estatística para cada uma das Supervisões Técnicas de Saúde 7 .

Se o IN S éuma ferramenta utilizada pel o serviço público para o acompanhamento das necessidades em saúde da população do município de São Paulo, orientando as ações e os serviços oferecidos, seria oportuno identificar se este indicador também coincide com as necessidades em saúde bucal, o que sustentaria a Área Técnica em Saúde Bucal para a importância de tomada de ações em conjunto.

Pretende-se, com este estudo, analisar a tendência do acesso aos serviços de saúde bucal, do perfil de saúde bucal e da necessidade de tratamento cirúrgico odontológico de crianças paulistanas de 5, 12 e 15 anos de idade em relação ao INS, considerando-se que esse índice poderá ser utilizado como subsídio técnico às decisões políticas de implantação de novos serviços em saúde.

\section{Métodos}

Trata-se um estudo de observação do tipo ecológico onde se utilizou dados agregados oriundos de dois estudos independentes realizados pela SM S-SP: 0 “Índice de N ecessidades em Saúde por Distrito Administrativo do M unicípio de São PauIo, 2008"5 e o "Levantamento Epidemiológico em SaúdeBucal da Cidade de São Paulo, 2008-2009"7.

\section{Índice de Necessidades em Saúde - INS}

Desde 2001 a Secretaria M unicipal da Saúdeda cidade de São Paulo, em parceria com a CEInfo, vem utilizando indicadores de saúde e estruturando um painel de monitoramento das condições de vida e saúde da população, o que possibilita analisar a situação dos serviços de saúde ofertados para cada um dos distritos administrativos da cidade. A construção do primeiro relatório foi em $2004^{8}$ e o índice, então chamado deĺndice-Saúde, tinha a finalidade de avaliar a equidade na distribuição dos serviçosnas 31 subprefeituras. Em 2005 foi editada uma nova versão com os dados evolutivos do biê nio 2004-2005. A terceira edição, publicada em 2008, apresentou a construção de um novo índice, onde foram agregados mais componentes de análise e que considerou, como espaço de observação e monitoramento, os 96 Distritos Administrativos da cidade de São Paulo, passando a ser denominado de Índice de N ecessidades em Saúde - IN S5. 
0 índice denecessidades em saúde (INS) editado em $2008^{5}$ foi construído a partir da agregação de 20 indicadores, subdivididos em componentes, que refletem aspectos prioritários da política de saúde, incluindo períodos etários e condições específicas individuais e ambientais que, no seu conjunto, sintetizam uma realidade que abrange condi ções de vida e de saúde 5 . São cinco os componentes: Crianças e Adolescentes; Gestantes; Adultos; I dosos e Doenças de N otificação Compulsória.

Para cada um dos componentes são considerados quatro indicadores, identificados como seguem: Crianças e Adolescentes (Proporção de crianças e adolescentes na população residente; Coeficientedemortalidadeinfantil; Coeficientede mortalidade por infecção respiratória aguda em menores de cinco anos; Proporção de gestantes adolescentes); Gestantes (Taxa de fertilidade total; Razão demortalidadematerna; Coeficientede incidência desífilis congênita eProporção de pré natal inadequado - menosdeseteconsultas); Adultos ( prevalência dehipertensão arterial; Prevalência de diabetes; Taxa de mortalidade por doenças crônicas relacionadasà hipertensão eTaxa demortalidadepor acidentes); I dosos (Proporção deidoso na população residente; M ortalidade precoce em idosos; Taxa de mortalidade de idosos por pneumonia; Taxa de mortalidade de idosos por acidentes); Doenças de notificação compulsória (Coeficientedeincidência por dengue; Coeficiente de incidência detuberculose pulmonar bacilífera; Coeficiente de incidência de leptospirose e Coeficiente deincidência demeningite).

A basepara a construção desteindicador sintético éa mesma do Índice de Desenvolvimento H umano (IDH), desenvolvido pela Organização Mundial da Saúde (OMS), por meio do Programa das Nações Unidas para o Desenvolvimento (PNUD) e pelo Instituto de Pesquisa Econômica A plicada (IPEA) ${ }^{10}$. O u seja, o IN S medea posição relativa de cada distrito administrativo à discrepância máxima apresentada por cada indicador. O INS de cada DA é cal culado para cada componente, sendo composto pela agregação dos valores relativos dos 20 indicadores previamente selecionados. O IN S sintético resulta da média dos valores obtidos para os indicadores dos cinco componentes 5 .

Para cada um dos indicadores foi calculada a posição ocupada pelo seu valor em relação à discrepância máxima. Os 96 Distritos Administrativos foram categorizados em três intervalos de igual valor definidos pela subdivisão da diferença entre o maior e o menor valor da cidade no índice final, classificando os DA em Baixa, M edia e Alta necessi dade em saúde.

Levantamento Epidemiológico em Saúde Bucal da Cidade de São Paulo,

2008-2009- LESB

Esse levantamento epidemiológico em saúde bucal, aprovado pelo Comitê de Ética em Pesquisa da SM S-SP, foi realizado em 1.371 escolas públicas e 374 privadas, no período de setembro a dezembro de 2008. 0 delineamento da amostra foi probabilístico por conglomerado em dois estágios. Na primeira etapa foram sorteadas as escolas públicas e as privadas, estratificadas por Supervisão Técnica de Saúde; na segunda etapa foram sorteados os escolares. Foram examinadas as condições dentárias de 5.557 crianças de 5 anos; 4.249 crianças de 12 anos e 1.566 jovens de 15 anos. Empregaram-se os índices ceod (média de dentes cariados, com extração indicada e obturados) para a dentição decídua e CPOD (média de dentes cariados, perdidos e obturados) para a dentição permanente, segundo metodologia recomendada pela $\mathrm{OM} \mathrm{S}^{7,11}$.

Os pais ou responsáveis preencheram um formulário para a caracterização sociodemográfica da população de estudo e sobre o acesso ao serviço de saúde bucal.

O relatório do levantamento informa os procedimentos de treinamento e calibração dos cirurgiões-dentistas que atuaram como examinadores e apresenta os dados de precisão inter-observador que garantem a confiabilidade; o valor encontrado para a estatística kappa de avaliação de condição de cárie dentária foi de 0,954 $(I C=0,94-0,96)^{7}$.

\section{Análise dos bancos de dados}

Os dados do INS foram apresentados pelos 96 DA da cidade de São Paulo e os do LESB pelas 25 STS. I sto posto, recalculou-seo I NS para cada uma das Supervisões de Saúde agregando-se os dados do IN S segundo os DA que compõem cada supervisão. Criou-se um novo banco de dadose as informações foram analisadas segundo os valores das 25 STS.

Considerando-se que o Índice de N ecessidades em Saúde(INS) foi desenvolvido para possibilitar a identificação de áreas e grupos populacionais a serem priorizados para a oferta de serviços de saúde na cidade de São Paulo, verificou-se a oferta de serviços e recursos humanos em saúde bucal. 
Correlacionou-se o INS com as medidas agregadas do LESB, estratificadas para as idades de 5, 12 e 15 anos e expressas pelas variáveis: porcentagem dos indivíduos que tiveram dificuldade no acesso ao serviço de saúde bucal (caracterizado pela proporção dos que nunca foram ou daqueles que foram há mais de três anos a uma consulta odontológica); média dos índices ceod (5 anos) e CPOD (12 e 15 anos); prevalência da necessidade de extração de dentes ea prevalência de crianças e jovens livres de cárie dentária.

Para a análise empregaram-se os testes estatísticos de covariância e o coeficiente de correlação de Pearson para identificar se houve correlação entre o INS e as variáveis de saúde bucal. A presentaram-se o modelo de regressão linear e os gráficos de dispersão.

\section{Resultados}

0 município de São Paulo, em seus 1.523 Km², tinha em 2007 uma população estimada em 10.886.518 habitantes ${ }^{12}$. A cidade está dividida em 96 Distritos Administrativos, que se agregam em cinco Coordenadorias Regionais de Saúde; cada uma delas possui problemas específicos e relevantes, que devem ser melhor compreendidos econsiderados para as estratégias de ação em saúde.

Os 96 DA foram categorizados em três intervalos, sendo que 19 deles apresentaram baixas necessidades de saúde; outros 47 foram classificados como tendo necessidades moderadas e 30 deles foram classificados como de alta necessidade em saúde. Os dados da classificação dos DA segundo as Coordenadorias Regionais de Saúde foram apresentados na Tabela 1.
Aszonas leste esul apresentaram, respectivamente, $70,6 \%$ e $60,0 \%$ dos seus distritos com alta necessidade; as regiões sudeste e norte tiveram $78,3 \%$ e $61,1 \%$ dos DA classificados como de média necessidade e a zona centro-oeste foi a única que apresentou a maioria $(47,8 \%)$ dos DA com baixa necessidade em saúde.

Em relação à capacidade física instalada, 0 município de São Paulo tinha 383 unidades de saúde com atendimento odontológico ambulatorial (US), onde trabal havam 1.355 cirurgiõesdentistas(CD) eoutros 872 profissionais da equipede saúde bucal (ESB), que desempenhavam as funções de Auxiliares de Saúde Bucal (ASB) ou Técnicos de Saúde Bucal (TSB). Calcularam-se as relações de CD e ESB por US e os dados, segundo as Coordenadorias Regionais de Saúde, que foram apresentados na Tabela 2.

Embora não incluídos no âmbito da atenção básica, mas fundamentais para que se busque a integralidade do sistema de saúde bucal, deacordo com dados do M inistério da Saúde, até maio de 2010 haviam sido habilitadosnacidadedeSão Paulo 22 Centros deEspecialidades 0 dontológicas, o que amplia a capacidade assistencial do município.

O INS pareceu acompanhar a disponibilidade de recursos humanos, sendo que a CRS Centro-Oeste, com menores necessidades em saúde, possuía a melhor relação cirurgião-dentista por Unidade de Saúde $(4,9)$.

A pior relação CD/US $(2,8)$ foi justamentena CRS Leste, que apresentou os piores INS e a maior proporção de DA classificados com alta necessidade em saúde (70,6\%).

A relação ESB/US foi homogênea (de 2,2 a $2,5)$ nas cinco Coordenadorias, entretanto, estes valores expressam uma relação desfavorável para

Tabela 1. Distribuição de frequência dos Distritos Administrativos (DA) segundo níveis do índice de Necessidades em Saúde (IN S) por Coordenadorias Regionais de Saúde. M unicípio de São Paulo, 2007.

\begin{tabular}{|c|c|c|c|c|c|c|c|c|}
\hline \multirow{3}{*}{$\begin{array}{l}\text { Coordenadoria } \\
\text { Regional de Saúde }\end{array}$} & \multicolumn{8}{|c|}{ Nível do INS - Índice de Necessidades em Saúde } \\
\hline & \multicolumn{2}{|c|}{$\begin{array}{l}\text { Baixa necessidade } \\
0,07401 \text { a } 0,20240\end{array}$} & \multicolumn{2}{|c|}{$\begin{array}{c}\text { Média necessidade } \\
0,20241 \text { a 0,33081 }\end{array}$} & \multicolumn{2}{|c|}{$\begin{array}{c}\text { Alta necessidade } \\
0,33082 \text { a } 0,45922\end{array}$} & \multicolumn{2}{|c|}{ Total } \\
\hline & $\mathrm{n} 0$ & $\%$ & $\mathrm{n} \cong$ & $\%$ & $\mathrm{n}^{0}$ & $\%$ & $\overline{\mathrm{n}} \underline{0}$ & $\%$ \\
\hline Centro-O este & 11 & 47,8 & 10 & 43,5 & 2 & 8,7 & 23 & 100,0 \\
\hline Leste & - & - & 5 & 29,4 & 12 & 70,6 & 17 & 100,0 \\
\hline Norte & 1 & 5,6 & 11 & 61,1 & 6 & 33,3 & 18 & 100,0 \\
\hline Sudeste & 4 & 17,3 & 18 & 78,3 & 1 & 4,4 & 23 & 100,0 \\
\hline Sul & 3 & 20,0 & 3 & 20,0 & 9 & 60,0 & 15 & 100,0 \\
\hline São Paulo & 19 & 19,8 & 47 & 49,0 & 30 & 31,1 & 96 & 100,0 \\
\hline
\end{tabular}

Fonte: São Paulo, 2008. 
Tabela 2. Número de Unidades de Saúde (US) que apresentam serviços de saúde bucal, número de cirurgiões-dentistas (CD) e Equipe de Saúde Bucal (ESB - Auxiliar em Saúde Bucal e Técnico em Saúde Bucal) e a Relação por Unidade de Saúde por Coordenadorias Regionais de Saúde. M unicípio de São Paulo, 2009.

\begin{tabular}{|c|c|c|c|c|c|}
\hline \multirow{2}{*}{$\begin{array}{c}\text { Coordenadoria Regional } \\
\text { de Saúde }\end{array}$} & \multirow{2}{*}{$\begin{array}{l}\text { Unidades } \\
\text { de Saúde }\end{array}$} & \multicolumn{2}{|c|}{ Cirurgião-dentista } & \multicolumn{2}{|c|}{ Equipe de Saúde Bucal } \\
\hline & & $\mathrm{n} \underline{0}$ & CD/US & $\mathrm{n} 0$ & $\mathrm{ESB} / \mathrm{US}$ \\
\hline Centro-Oeste & 34 & 167 & 4,9 & 84 & 2,5 \\
\hline Leste & 101 & 284 & 2,8 & 223 & 2,2 \\
\hline Norte & 77 & 267 & 3,5 & 170 & 2,2 \\
\hline Sudeste & 89 & 353 & 3,9 & 200 & 2,2 \\
\hline Sul & 82 & 284 & 3,5 & 195 & 2,4 \\
\hline São Paulo & 383 & 1355 & 3,5 & 872 & 2,3 \\
\hline
\end{tabular}

o melhor proveito de recursos humanos auxiliares no desenvolvimento das atividades clínicas e de ações coletivas, o que pode levar à baixa produtividade das ações de saúde bucal.

Dados agregados dos DA que compõem as 25 Supervisões Técnicas de Saúde, referentes ao INS, à porcentagem de crianças que tiveram dificuldade no acesso aos serviços de saúde bucal eà porcentagem decrianças com necessi dade de extração dentária, estratificados pelas idades, foram apresentados na Tabela 3.

As maiores dificuldades no acesso à consulta odontológica foram verificadas nas crianças de 5 anos de idade. Com exceção da STS de Ermelino $M$ atarazzo, todas as outras da CRS Leste e as da CRS Sul apresentaram mais de 30,0\% das crianças aos 12 anos com al guma dificuldade no acesso ao CD. Deuma maneira geral, a dificuldadeno acesso à consulta odontológica aos 15 anos de idade diminuiu em relação aos 12 anos de idade, visto que, em 16 STS a porcentagem dejovensque nunca foram ao $C D$ ou que foram há mais detrês anos foi menor do que aos 12 anos de idade.

A dificuldade no acesso à consulta odontológica na infância pode gerar um agravamento na carga de doença, o que ocasiona a necessi dade de extração dentária. Aos 15 anos deidade 7,3\% dos jovens necessitavam de al guma intervenção mutiladora, principalmente os da CRS Leste.

A Tabela 4 apresenta dados do Coeficiente de Covariância e do Coeficiente de Correlação de Pearson em relação ao INS e às variáveis de saúde bucal. Há correlações positivas, expressas nas três idades, entre alta necessidade em saúde e a dificuldade deacesso aos serviços de saúde bucal, maior média dosíndices ceod eCPOD e maiores percentuais de necessidades de exodontias.
Onde há menores necessidades em saúde há uma maior prevalência decrianças livres de cárie dentária, por isso os valores dos coeficientes de covariância e de correlação de Pearson foram negativos.

A Figura 1 ilustra os gráficos de dispersão relacionados às variáveis de saúde bucal eo Índice de Necessidades em Saúde. Observa-se que, com 0 aumento do INS aumentaram-se também as dificuldades no acesso, os índices de cárie para a dentição decídua e permanente e a necessidade deextração dentária. A relação foi inversa para a porcentagem de crianças livres de cárie. Para todas as variáveis a inclinação das retas foi mais tênue aos 15 anos de idade.

\section{Discussão}

Sabe-se que estudos ecológi cos baseados em dados secundários oriundos de distintas fontes dificultam o controle de possíveis efeitos de confusão de fatores não modelados no nível de agregação do estudo, além de impossibilitar a inferência, para o nível individual, dos resultados obtidos ao nível populacional ${ }^{13}$.

M as optou-se por um estudo do tipo ecológico, poisse pretendia explicar os efeitos contextuais sobre a necessidade de serviços em saúde e os indicadores de saúde bucal para a contribuição na adoção de políticas para o cuidado com a saúde bucal.

Admite-se que o uso dos serviços de saúde, inclusive os odontológicos, deve ser considerado em três dimensões: a capacitação, a predisposição ea necessidade, conforme proposto por Andersen e N ewman ${ }^{14}$. 
Tabela 3. Índice de Necessidades em Saúde, Porcentagem de crianças com dificuldade de acesso aos serviços de saúde bucal e Porcentagem de crianças com necessidade de extração dentária segundo idade e Supervisões Técnicas de Saúde. M unicípio de São Paulo, 2008.

\begin{tabular}{|c|c|c|c|c|c|c|c|}
\hline \multirow{3}{*}{$\begin{array}{c}\text { Supervisão Técnica } \\
\text { de Saúde }\end{array}$} & \multirow{3}{*}{ INS } & \multicolumn{3}{|c|}{$\begin{array}{l}\text { Crianças que nunca foram ou } \\
\text { foram ao CD há mais de } 3 \text { anos }\end{array}$} & \multirow{2}{*}{\multicolumn{3}{|c|}{$\begin{array}{c}\begin{array}{c}\text { Necessidade de } \\
\text { extração dentária }\end{array} \\
\text { Idade (anos) }\end{array}$}} \\
\hline & & \multicolumn{3}{|c|}{ I dade (anos) } & & & \\
\hline & & 5 & 12 & 15 & 5 & 12 & 15 \\
\hline \multicolumn{8}{|l|}{ Centro-Oeste } \\
\hline Butantã & 0,24608 & 50,8 & 27,9 & 20,2 & 2,5 & 5,4 & 8,4 \\
\hline Lapa & 0,16316 & 15,5 & 8,7 & 2,8 & 0,9 & - & \\
\hline Sé & 0,23886 & 49,9 & 28,2 & 20,0 & 3,3 & 1,3 & 6,7 \\
\hline \multicolumn{8}{|l|}{ Leste } \\
\hline Cid. Tiradentes & 0,35700 & 44,2 & 32,0 & 38,8 & 5,2 & 6,1 & 12,2 \\
\hline Erm. M atarazzo & 0,30652 & 53,2 & 25,5 & 27,9 & 3,2 & 2,3 & 8,6 \\
\hline Guaianases & 0,40122 & 65,6 & 37,9 & 44,8 & 4,8 & 8,2 & 19,0 \\
\hline Itaim Paulista & 0,38293 & 58,8 & 44,8 & 30,6 & 7,1 & 3,8 & 10,2 \\
\hline Itaquera & 0,31650 & 55,5 & 32,9 & 34,3 & 2,5 & 4,8 & 4,0 \\
\hline São Mateus & 0,34651 & 37,3 & 34,3 & 27,1 & 6,2 & 8,0 & 9,4 \\
\hline São Miguel & 0,35423 & 53,8 & 34,3 & 18,3 & 5,4 & 4,2 & 1,6 \\
\hline \multicolumn{8}{|l|}{ Norte } \\
\hline Casa. Verde & 0,30214 & 47,7 & 24,6 & 17,6 & 6,7 & 3,4 & \\
\hline Freguesia do Ó & 0,33724 & 51,8 & 35,9 & 39,6 & 4,2 & 5,6 & 6,6 \\
\hline Pirituba & 0,29548 & 46,7 & 39,1 & 29,6 & 4,8 & 6,2 & 9,9 \\
\hline Santana & 0,25456 & 49,0 & 18,6 & 15,9 & 3,4 & 2,1 & \\
\hline V.M aria & 0,31491 & 18,4 & 6,1 & 1,4 & 4,0 & 5,5 & 5,7 \\
\hline \multicolumn{8}{|l|}{ Sudeste } \\
\hline Mooca & 0,25761 & 32,7 & 20,9 & 25,4 & 3,9 & 1,0 & 5,1 \\
\hline Ipiranga & 0,25144 & 39,6 & 17,8 & 27,8 & 5,1 & 0,8 & 3,8 \\
\hline Penha & 0,28413 & 41,1 & 22,2 & 32,6 & 2,6 & 5,1 & 4,7 \\
\hline Vila Mariana & 0,15173 & 32,9 & 12,2 & 2,3 & 3,9 & 0,4 & 2,3 \\
\hline Vila Prudente & 0,26934 & 55,9 & 41,9 & 38,0 & 4,7 & 3,6 & 12,7 \\
\hline \multicolumn{8}{|l|}{ Sul } \\
\hline Campo Limpo & 0,32055 & 44,5 & 30,0 & 26,7 & 5,0 & 4,3 & 4,6 \\
\hline Capela Socorro & 0,32158 & 48,7 & 32,7 & 47,7 & 3,9 & 2,6 & 4,2 \\
\hline M'Boi Mirim & 0,39507 & 42,6 & 36,8 & 27,6 & 8,3 & 4,2 & 5,1 \\
\hline Parelheiros & 0,39748 & 64,3 & 49,6 & 16,0 & 5,3 & 5,8 & 8,0 \\
\hline Santo Amaro & 0,25756 & 44,7 & 38,4 & 36,3 & 6,2 & 7,5 & 8,5 \\
\hline
\end{tabular}

Tabela 4. Coeficiente de Covariância, Coeficiente de Correlação de Pearson e Regressão linear entre Índice de N ecessidades em Saúde (INS) e condições de saúde bucal, segundo idade. M unicípio de São Paulo, 2008.

\begin{tabular}{lcccccc}
\hline \multicolumn{1}{c}{ Condições de Saúde Bucal } & I dades & Covariância & CC Pearson & $r^{2}$ & $r_{\text {ajust. }}^{2}$ & $p$ \\
\hline Dificuldade de acesso aos serviços & 5 & 0,426 & 0,566 & 0,31 & 0,28 & 0,003 \\
de saúde bucal & 12 & 0,468 & 0,675 & 0,45 & 0,43 & 0,000 \\
& 15 & 0,376 & 0,482 & 0,23 & 0,19 & 0,015 \\
Média dos índices ceod e CPOD & 5 & 0,014 & 0,551 & 0,30 & 0,27 & 0,004 \\
& 12 & 0,011 & 0,428 & 0,18 & 0,14 & 0,033 \\
& 15 & 0,014 & 0,321 & 0,10 & 0,06 & 0,117 \\
Porcentagem de crianças com & 5 & 0,062 & 0,600 & 0,35 & 0,33 & 0,002 \\
necessidades de extração dentária & 12 & 0,094 & 0,638 & 0,40 & 0,38 & 0,001 \\
& 15 & 0,130 & 0,466 & 0,21 & 0,18 & 0,019 \\
Prevalência de crianças livres de & 5 & $-0,330$ & $-0,419$ & 0,38 & 0,35 & 0,001 \\
cárie dentária & 12 & $-0,418$ & $-0,591$ & 0,34 & 0,32 & 0,002 \\
& 15 & $-0,422$ & $-0,558$ & 0,31 & 0,28 & 0,004 \\
\hline
\end{tabular}



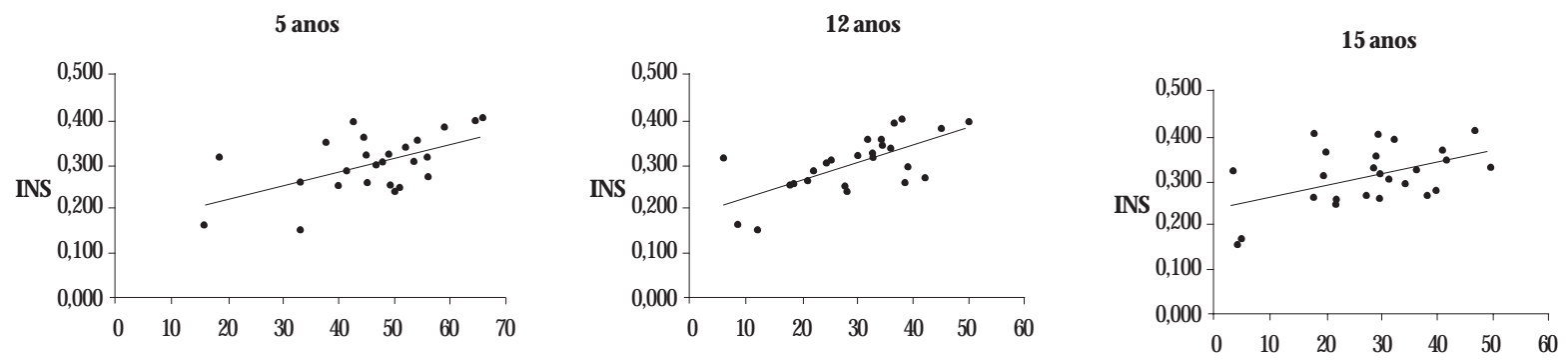

Porcentagem de crianças com dificuldade de acesso aos serviços de saúde bucal
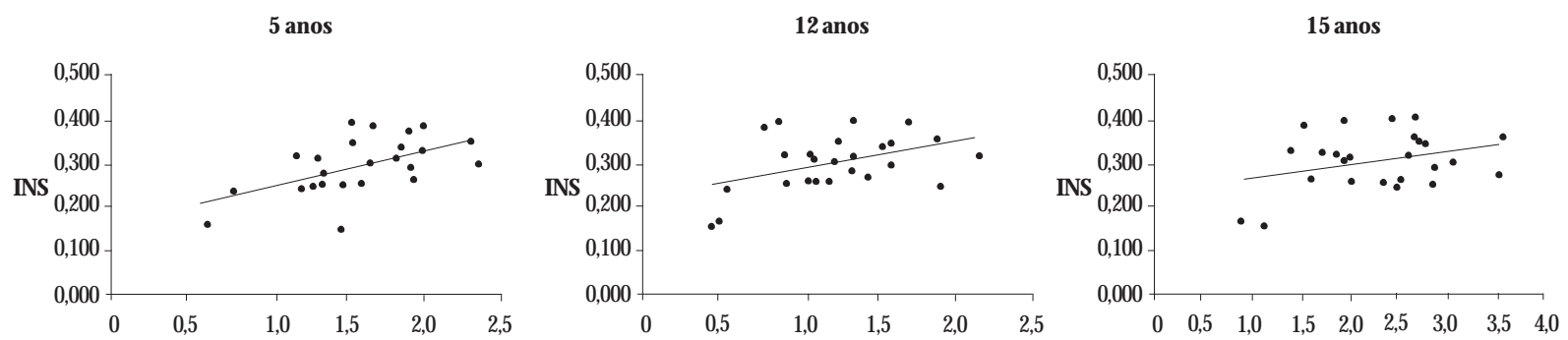

Média do índiceCPO-D
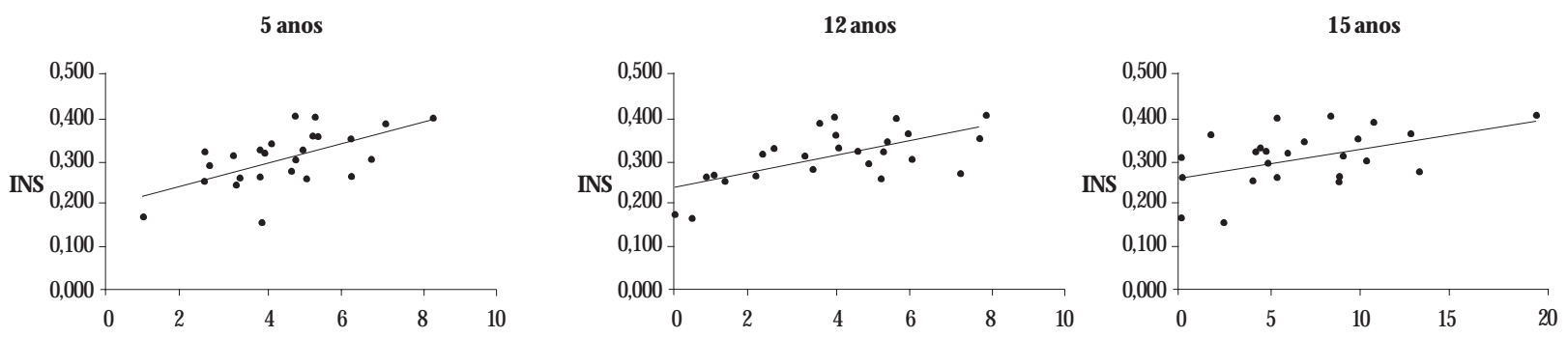

Porcentagem de crianças com necessidade de extração dentária
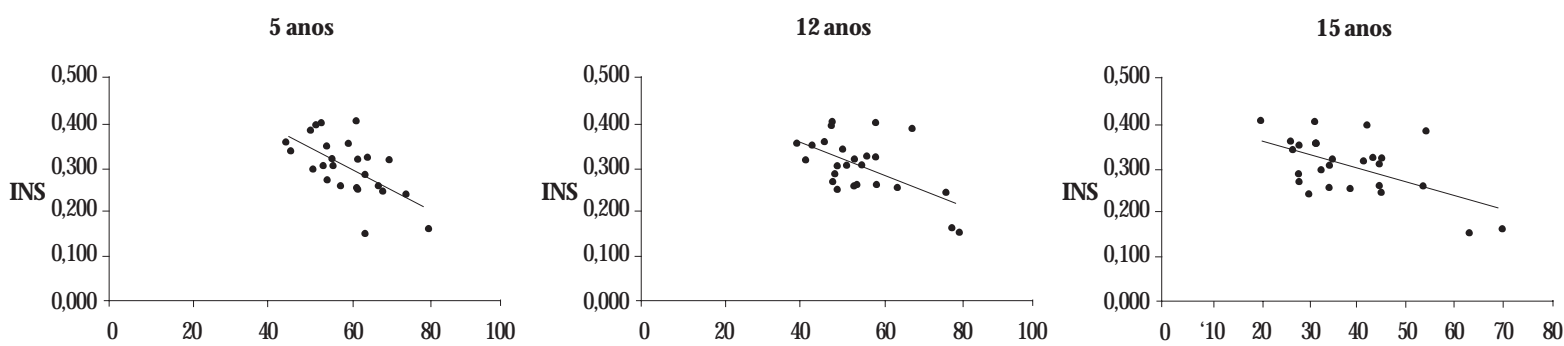

Porcentagem de crianças livres de cárie

Figura 1. Diagrama de dispersão do INS (Índice de N ecessidade em Saúde) e dificuldade de acesso aos serviços de saúde bucal, média do índice ceod e CPOD, porcentagem de crianças com necessidades de extração dentária e a prevalência de crianças livres de cárie dentária, nas idades de 5, 12 e 15 anos na cidade de São Paulo, 2008. 
A primeira refere-se ao fato de o indivíduo procurar e receber atendimento, tendo como variáveis, entre outras, a condição econômica e a oferta de serviços; a predisposição está relacionada a características individuais queaumentem as chances de procura pelos serviços, como idade e hábitos nocivos e a necessidade refere-se à percepção dos indivíduos sobre seu estado de saúdee a própria condição desaúde, medida pelo seu avesso, ou seja, a doença.

Pioresíndices denecessidades em saúde são o reflexo das privações sociais aos quais estão expostas a população e os índices de saúde bucal tendem a ser piores em locais com privações sociais ${ }^{15}$. N esteestudo, osindicadores de saúdebucal acompanharam essa tendência (Figura 1).

Até 2005, eram considerados como indicadores de saúde bucal pelo M inistério da Saúdea cobertura de primeira consulta odontológica e a razão entre procedimentos odontológicos coletivos ea população de 0 a 14 anos. Como indicador complementar, sugeria-se a proporção de exodontias em relação às ações odontológicas básicas individuais.

A partir de 2006, com a Portaria $n=493$, de 10 de março de $2006^{16}$, reiterada pela Portaria $\mathrm{n}$ 은 325 , de 21 de fevereiro de $2008^{17}$, passaram a ser considerados indicadores da atenção básica em saúde bucal a cobertura de primeira consulta odontológica programática ea cobertura da ação coletiva escovação dental supervisionada.

Deve-se ressaltar a fragilidade do indicador relacionado à primeira consulta odontológica, pois, embora a intenção seja medir a porcentagem da população que teve acesso ao cirurgião-dentista do serviço público, muitas vezes esse val or ésuperestimado, pois se incorporam ao indicador os atendimentos de emergência, realizados na primeira consulta. Para a odontologia, isso não representa intervenção paraa adequada recuperação e controle das doenças bucais e, para os serviços, não demonstra resolutividade, pois o pacientenão retorna para completar o tratamento.

Neste trabalho 0 acesso foi medido por um questionamento direto aos responsáveis pelas crianças quanto ao tempo desde a última consulta odontológica, admitindo-se como uma dificuldadeno acesso o fato denunca ter consultado o profissional ou têlo feito há mais de 3 anos. Não se questionou quais as justificativas desse acesso restrito, se pelo fato denão terem necessitado ou porque os paisjulgaram não ser importante consultar 0 cirurgião-dentista. 0 que se percebeu foi que 0 pior acesso se deu aos 5 anos de idade, fato esteque também pode estar relacionado à pouca oferta de serviços para essa população que, pela dificuldade de colaborar com o tratamento, inerenteà idade ${ }^{18}$, requer atuação de profissional especializado.

Gillcrist et al. ${ }^{19}$ indicam que 0 acesso aos serviços é diferenciado para os diferentes grupos sociais, sendo que crianças provenientes de famílias de baixo poder aquisitivo tiveram menor possibilidade de acesso aos serviços de saúde.

Estudos sobre o uso dos serviços de saúde pela população adolescente, adulta eidosa apontam para o fato de que ele émenor quanto piores os indicadores socioeconômicos e de condições de saúde ${ }^{20-25}$.

O uso de serviços de saúde é também função das necessidades e do comportamento dos indivíduos diante dos seus problemas de saúde ${ }^{21}$, ou seja, sua predisposição.

A cárie é uma doença multifatorial e vários são os fatores que determinam o seu surgimento, inclusive os de natureza socioambiental. Embora mudanças socioeconômicas tenham sido mais relevantes para a redução da cárie do que a oferta de serviços odontológicos ${ }^{26}$, sendo esta doença um dos principais problemas de saúde pública no país, o uso dos serviços odontológicos passa a ser de fundamental importância para o seu controle. Quanto aos aspectos relativos à necessidade, servem de referência o índice ceod/CPOD ea necessidade de tratamento para a cárie.

M aior prevalência de cárie ou menor porcentagem decrianças livres dela, em região da cidade com alta e moderada necessidades em saúde, podem ser reflexo dessa rede de determinantes no processo saúde-doença.

Em estudo sobre adolescentes do estado de São Paulo, a utilização dos serviços, em relação à cárie, seassociou aos maioresíndices ceod/CPOD. No entanto, a saúde gengival dos jovens foi meIhor para aqueles que mais procuraram os serviços odontológicos, independente das características sociodemográficas in dividuais e contextuai $s^{27}$.

Estudo ecológico de associação entre indicadores de atenção básica e a provisão de serviços odontológicos nos municípios de Santa Catarina evidenciou quemunicípios com piores condições socioeconômicas apresentaram maior proporção de exodontias ${ }^{28}$.

Conforme aumentou a razão de profissionais cadastrados no SUS em relação à população dos municípios de Santa Catarina, aumentaram a cobertura da primeira consulta odontológica e a proporção de procedimentos coletivos, com diminuiç̧ão das exodontias ${ }^{26}$.

Estudo realizado nos municípios de estado do Paraná demonstrou haver uma correlação positi- 
va entreo índice CPOD eo número deconsultórios disponíveis no serviço. Municípios com piores indicadores sociais foram os que ofereceram uma maior cobertura de serviços públicos à população 29,30 , indicando uma política positiva de redirecionamento de recursos financeiros etecnológicos segundo necessidades sociais e epidemiológicas.

Tem havido, no setor público, o direcionamento dosrecursoshumanosemateriais deacordo com as necessidades da população. No estado de São Paulo, quanto piores os indicadores sociais, maior foi o número de cirurgiões-dentistas e auxiliares, bem como de equipamentos, no serviço público municipal ${ }^{31}$.

A oferta na disponibilidade de recursos humanos nas Coordenadorias Regionais de Saúde seguiu a tendência dos indicadores de necessidades em saúdee das variáveis de saúde bucal estudadas.

Neste trabalho, observou-se que as regiões com maior necessidade em saúde também tiveram uma menor relação dentista por Unidadede Saúde. A incorporação de equipe auxiliar mostrou-se baixa em todas as regiões. Estes dados expressam uma relação desigual quanto aos recursos humanos adequados para o desenvolvimento das atividades clinicas e deações coletivas, o que pode gerar baixa produtividade das ações de saúde bucal.

É desejável que haja profissionais em quantidade adequada para o controle das enfermidades bucais, mas há que se refletir sobre sua interferência nos índices de saúde bucal.

A relevância deestudos ecológicosna política eno planejamento sanitário se faz por identificar áreas com maiores problemas sociosanitários, que devem ser consideradas para a elaboração de estratégias ajustadas à diversidade dos problemas. M édias favoráveis de saúde bucal podem encobrir níveis preocupantes de cárie dentária em grupos populacionais específicos ${ }^{32}$.

Neste estudo, os indicadores de saúde bucal e de acesso aos serviços acompanharam o INS. Reforça-se, portanto, a necessidade de se priorizar as áreas de privação social na cidade de São Paulo para melhorar a condição de saúde bucal da população e minimizar os efeitos da desigual dade.

\section{Colaboradores}

SR Junqueira participou do desenho e concepção do estudo, interpretação dos dados e redação final do artigo. AC Frias trabalhou no desenho e concepção do estudo, na análise e interpretação dos dados. C Zilbovicius e M E Araujo participaram na concepção e na redação final do artigo.

\section{Referências}

1. Antunes JLF, Peres MA, M ello TRC, Waldman EA. Multilevel assessment of determinants of dental caries experience in Brazil. Community Dent Oral Epidemiol 2006; 34(2):146-152.

2. Brasil. M inistério da Saúde. Coordenação Nacional de Saúde Bucal. Projeto SB Brasil 2003: condições de saúde bucal da população brasileira, 20022003, resultados principais. Brasília: Ministério da Saúde; 2004.

3. Narvai PC, Frazão P, Roncalli AG, Antunes JLF. Cárie dentária no Brasil: declínio, iniqüidade e exclusão social. Rev Panam Salud Publica 2006; 19(6):385-393.

4. Moreira RF, Nico LS, Tomita NE. A relação entre o espaço e a saúde bucal coletiva: por uma epidemiologia georreferenciada. Cien Saude Colet 2007; 12(1):275-84.

5. São Paulo. Prefeitura do M unicípio de São Paulo. Secretaria M unicipal da Saúde (SM S). Coordenação de Epidemiologia e Informação - CEInfo. Índice de Necessidades em Saúde por Distrito Administrativo do M unicípio de São Paulo. 3a edição. São Paulo: Secretaria M unicipal da Saúde (SM S); 2008.

6. Moreira TP, Nations M K, Alves M SCF. Dentes da desigualdade: marcas bucais da experiência vivida na pobreza pela comunidade do Dendê, Fortaleza, Ceará, Brasil. Cad Saude Publica 2007; 23(6):13831392. 
7. São Paulo. Prefeitura do Município de São Paulo. Secretaria M unicipal da Saúde. Coordenação da Atenção Básica. Área Técnica de Saúde Bucal. Levantamento Epidemiológico em Saúde Bucal. Cidade de São Paulo, 2008-2009. Resumo da Primeira Fase: Crianças e Adolescentes. São Paulo: Coordenação da Atenção Básica - Área Técnica de Saúde Bucal; 2009.

8. São Paulo. Prefeitura do M unicípio de São Paulo. Secretaria M unicipal da Saúde (SM S). Coordenação de Epidemiologia e Informação - CEInfo. Painel de monitoramento das condições de vida e saúde e da situação dos serviços de saúde - Avaliação da equidade: Índice-Saúde 2004 por Subprefeitura e tendência nos últimos quatro anos. São Paulo: Secretaria M unicipal da Saúde (SM S); 2005.

9. São Paulo. Prefeitura do M unicípio de São Paulo. Secretaria M unicipal da Saúde (SM S). Coordenação de Epidemiologia e Informação - CEInfo. Painel de monitoramento das condições de vida e saúde e da situação dos serviços de saúde - Avaliação da equidade: Índice-Saúde 2005 por Subprefeitura e tendência nos últimos anos. São Paulo: Secretaria M unicipal da Saúde (SM S); 2006.

10. Programa das Nações Unidas para o Desenvolvimento (PNUD). Instituto de Pesquisa Econômica Aplicada. Relatório do Desenvolvimento Humano no Brasil. Brasília: Fundação João Pinheiro; 2003.

11. World Health Organization (WHO). Oral health surveys: basic methods. $4^{\text {th }}$. ed. Geneva: World Health Organization (WHO); 1997.

12. Brasil. Instituto Brasileiro de Geografia e Estatística (IBGE). Cidades@ [site na Internet]. [acessado 2009 nov 10]. Disponível em: http://www.ibge.gov.br/cidadesat/topwindow.htm?1.

13. Peres MA, Antunes JLF. 0 método epidemiológico de investigação e sua contribuição para a saúde bucal. In: Crivello Júnior 0 , organizador. Fundamentos de Odontologia. Antunes JLF, Peres MA editores. Epidemiologia da saúde bucal. Rio de Janeiro: Guanabara Koogan, 2006. p.2-18.

14. Andersen R, Newman JF. Societal and individual determinants of medical care utilization in the United States. M ilbank M em Fund Quar 1973; 51(1):95124.

15. Frias AC, Antunes JLF, Junqueira SR, Narvai PC. Determinantes individuais e contextuais da prevalência de cárie dentária não tratada no Brasil. Rev Panam Salud Publica 2007; (22)4:279-285.

16. Brasil. Ministério da Saúde. Portaria no 493, de 10 de março de 2006. Aprova a relação de Indicadores da Atenção Básica - 2006, cujos indicadores deverão ser pactuados entre municípios, estados e M inistério da Saúde. Diário Oficial da União 2006; 13 mar.

17. Brasil. M inistério da Saúde (M S). Portaria no 325, de 21 de fevereiro de 2008. Estabelece prioridades, objetivos e metas do Pacto pala Vida para 2008, os indicadores de monitoramento e avaliação do Pacto pela Saúde e as orientações, prazos e diretrizes para a sua pactuação. Diário 0 ficial da União 2008; 13 mar.

18. Ferreira JMS, Aragão AKR, Colares V. Técnicas de Controle do Comportamento do Paciente Infantil: Revisão de Literatura. Pesq Bras 0 dontoped Clin Integr 2009; 9(2):247-251
19. Gillcrist JA, Brumley DE, Blackford JU. Community socioeconomic status and children's dental health. J Am Dent Assoc 2001; 132:213-222.

20. São Paulo. Fundação Sistema Estadual de Análise de Dados. Pesquisa de condições de vida na região metropolitana de São Paulo: saúde. São Paulo: SEADE; 1992.

21. Travassos C, Vaacava F, Fernandes C, Almeida CM . Desigualdades geográficas e sociais na utilização de serviços de saúde no Brasil. Cien Saude Colet 2000; 5(1):133-149.

22. Neri M, Wagner S. Desigualdade social e saúde no Brasil. Cad Saude Publica 2002; 18(Supl.):77-87.

23. Claro LBL, M arch C, M arcarebhas M TM, Castro $I A B$, Rosa M LG. Adolescentes e suas relações com serviços de saúde: estudo transversal em escolares de Niterói, Rio de Janeiro, Brasil. Cad Saude Publica 2006; 22(8):1565-1574.

24. Louvison MCP; Lebrão ML, Duarte YAO, Santos JLF, Malik AM, Almeida ES. Desigualdades no uso e acesso aos serviços de saúde entre idosos do município de São Paulo. Rev Saude Publica 2008; 42(4): 733-740.

25. Dias-da-Costa JS, Presser AD, Zanolla AF, Ferreira DG, Perozzo G, Freitas IBA, Portolan LT, Tavares RT, Olinto MTA, Pattussi MP. Utilização dos serviços ambulatoriais por mulheres: estudo de base populacional no Sul do Brasil. Cad Saude Publica 2008; 24(12):2843-2851.

26. Nadanovsky P, Sheiham A. Relative contribution of dental services to the changer in caries levels of 12 year-old children in 18 industrialized countries in the 1970s and early 1980s. Community Dent Oral Epidemiol 1995; 23(6):331-339.

27. Antunes JLF, Peres MA, Frias AC, Crosato EM, Biazevic MGH. Saúde gengival de adolescentes e a utilização de serviços odontológicos, Estado de São Paulo. Rev Saude Publica 2008; 42(2):191-199.

28. Fernandes LS, Peres MA. Associação entre atenção básica em saúde bucal e indicadores socioeconômicos municipais. Rev Saude Publica 2005; 39(6):390396.

29. Baldani M H, Vasconcelos AGG, Antunes JLF. Associação do índice CPO-D com indicadores sócioeconômicos e de provisão de serviços odontológicos no estado do Paraná, Brasil. Cad Saude Publica 2004; 20(1):143-152.

30. Baldani MH, Almeida EA, Antunes JLF. Equidade e provisão de serviços públicos odontológicos no estado do Paraná, Brasil. Rev Saude Publica 2009; 43(1):446-454.

31. Junqueira SR, Araújo ME, Antunes JLF, Narvai PC. Indicadores socioeconômicos e recursos odontológicos em municípios do estado de São Paulo, Brasil, no final do século XX. Epidemiologia e Serviços de Saúde 2006; 15(4):41-53.

32. Lucas SD, Portela MC, Mendonça LL. Variações no nível de cárie dentária entre crianças de 5 e 12 anos em M inas Gerais, Brasil. Cad Saude Publica 2005; 21(1):55-63.

Artigo apresentado em 27/08/2010

Aprovado em 22/11/2010

Versão final apresentada em 12/12/2010 